\title{
Trigger low-voltage devices electromechanical apparatus
}

\author{
$N Y u$ Zaitsev$^{1}, Y u M$ Zaitsev $^{1}, E V$ Mikhailova $^{2}, D S$ Sazanov $^{1}$ and $G P$ Svintsov $^{1}$ \\ ${ }^{1}$ Federal State Budgetary Educational Institution of Higher Education "Chuvash State University named after I.N. Ulyanov", \\ Cheboksary, Russia. \\ ${ }^{2}$ Federal State Budgetary Educational Institution of Higher Education " Kazan State Energy University ", Kazan, Russia
}

\begin{abstract}
Based on the analysis of the existing classification of low-voltage electromechanical devices, it was proposed, on the basis of the functions performed, to unite into the group of electromagnetic mechanisms the indicating relays, vacuum contactors, time relays with clockwork, and executive bodies of circuit breaker releases. The definition of launching devices is given, the main feature of which is a mechanical characteristic, the change of which, with appropriate coordination with the traction characteristic of an electromagnet, can lead to an improvement of the most important characteristics of these devices, such as power consumption and resistance to mechanical influencing factors. On the example of the calculation of the index relay, it is shown and experimentally confirmed that a change in the initial force of the mechanical characteristic and its rigidity leads to a decrease in power consumption while maintaining shock stability.
\end{abstract}

\section{Introduction}

In the literature $[1,2]$, low-voltage electromechanical devices (EMA) are classified according to a number of signs.

Selected [3] is a subclass of power electromagnetic mechanisms (EMM), which, according to its functional purpose, is divided into three characteristic groups: drive, holding and push.

However, in our opinion, it is expedient to single out a group from the whole variety of EMM, which, according to its functions, does not quite fit into the classification given. This group includes EMM indicating relays, a time relay with a clockwork, vacuum contactors and circuit breakers (AB), in which the EMM performs the function of lowering manually actuated or automatically actuating units of electrical apparatus.

To clarify the functional purpose of the EMM of this group, we consider the work cycles of the corresponding EMA.

Pointing relays RU-21, Rapu-12M. The cocking of the contact system (CS) and the pointing mechanism (PM) of the pointing relay is made manually. The winding of the electromagnet (EM) is de-energized and its anchor is in the initial position (initial gap). The CS and the PM are in the cocked position due to a special protrusion on the disk of PM resting on the anchor of the EMM.

When voltage (current) is applied to the EM winding, it triggers, the anchor moves to the end position (final gap) and, at a certain gap (trigger gap), the special protrusion on the disk of PM goes out of engagement with the anchor of EM and PM triggered.
Time relay with clockwork series RV-100. When the supply voltage is disconnected, the clock mechanism (FM) is cocked by the spring and is held by it in the cocked state.

When energized, the EM triggers and releases the FM, thereby providing the FM descent function. World Cup counts down the time delay.

When the tension is removed, the spring returns the armature EM to the initial position and cocks the FM, preparing it for the next actuation.

Vacuum contactor KV-1. When the voltage is removed from the winding of the electromagnet, the contacts of the vacuum chamber are open by a specially provided spring.

When voltage is applied to the winding, the armature EM compresses the spring and releases it from the action on the vacuum chamber; the contacts are closed due to the difference in atmospheric pressure and inside the chamber, that is, the EM descends the contacts of the vacuum chamber.

Automatic switches. The mechanism of free tripping (MCP) and the closure of contacts is carried out manually.

In the event of a short-circuit current, the release of a pre-charged MCP is carried out by an electromagnetic release.

The executive units of electronic and microprocessor-based AV releases most often, in particular [4], are implemented as electromagnetic mechanisms that descend the MCP after performing a certain algorithm (forming an inverse ampere characteristic, performing the function of a minimum release) by the electronic part of the release.

\footnotetext{
* Corresponding author: pvn.chuvsu@ yandex.ru
} 
It should be noted that in AV the thermo-bimetallic release also performs the function of the release of the MCP, since with the appearance of an overload current the thermal release begins to bend. After some time, the TR enters into mechanical interaction with the MCP and, at the moments of equality of the force developed by it, with the force of counteraction of the MCP, ensures the activation of the $\mathrm{AV}$ (descent of the MCP).

Thus, for functional purposes, you can select a characteristic group of devices, as part of certain EA, which can be called EA trigger devices (SUEA).

Definition: the trigger should be called devices intended for the descent, pre-cocked manually or automatically the executive bodies of the EA (contacts, index (PM), clockwork mechanisms, etc.).

A common feature that unites this group of EMMs is their mechanical characteristic, which, unlike EM-driven relays and contactors, has the form of a straight line, due to the action of the return spring only, which has a significant impact on the design methodology and performance.

The nature of the mechanical forces in the EA with the release devices allows, by varying the parameters of the return spring, to influence (in the direction of improvement) such important characteristics of the EA as power consumption, vibration and impact resistance.

The illustration of this position is shown on the example of the relay REPU-12M produced by LLC VNIIR-Promelektro, Cheboksary. Compared with existing analogues RU-21 (JSC "ChEAZ", Cheboksary) and REU-11 (JSC "BETZ", Bershad, Ukraine), the power consumption of the relay REPU-12M with current windings before accepting our proposals is slightly higher than the analogs, and was 0.35 watts versus 0.25 watts. To reduce the power consumption of the winding of the relay REPU-12M, it is necessary to reduce [1] the magnetomotive force (MDS) of the operation of Fop of this relay. Moreover, if the rigidity of the mechanical characteristics of the relay does not change, the resistance of the relay to shock and vibration loads will decrease. To maintain the resistance to mechanical stress, you can increase the rigidity of the mechanical characteristics [5]. For experimental verification, two prototypes of the relay REPU-12M were manufactured with mechanical characteristics of different stiffness, the windings of which provide $\mathrm{Fop}=125 \mathrm{~A}$ and the power consumption at nominal currents was checked, as well as comparative tests of the impact resistance of the experimental and serial samples. After the manufacturer accepted our proposals, the meaning of which was to reduce the spring force at the initial gap and increase its rigidity, the guaranteed power consumption was brought to the level $(0.25 \mathrm{~W})$ of analogues, thereby ensuring competitiveness.

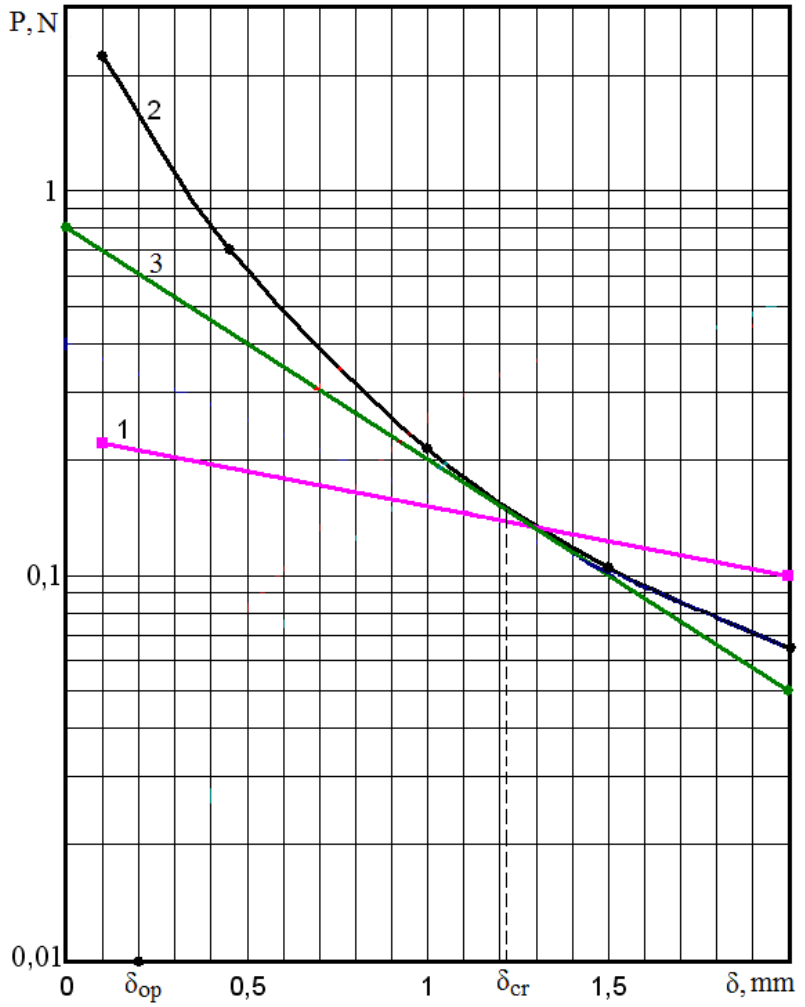

Fig. 1.

The figure shows the calculated traction characteristic of the electromagnet and the mechanical characteristic agreed with it. The calculations were carried out according to the method [1]. In this case, the following notation for the above dependencies:

1 - the mechanical characteristic of a serial relay with a hardness of $0,063 \mathrm{~N} / \mathrm{mm}$, at which the MDS of operation is equal to $F_{\text {op }}=238 \mathrm{~A}$ and the rated power consumption of the $P_{\text {rat }}=0,35 \mathrm{~W}$;

2 - traction characteristic of an electromagnet with a magnetomobile actuation force $F_{\text {op }}=125 \mathrm{~A}$;

3 - calculated mechanical characteristic with a hardness of $0.375 \mathrm{~N} / \mathrm{mm}$, in which the MDS of operation is $F_{\text {op }}=125 \mathrm{~A}$ and the power consumed by the electromagnet is not more than $0,25 \mathrm{~W}$;

$\delta_{\text {op }}$ - gap operation of the UP between the anchor and the core;

$\delta_{\text {cr }}$ - critical gap between the armature and the core, in which the traction characteristic at $F_{\text {op }}=125 \mathrm{~A}$ has one common point with the mechanical characteristic.

The tests of the relays on the effects of mechanical shocks were carried out with rectangular shock pulses with a duration of $20 \mathrm{~ms}$ and 20 shocks with successive changes in acceleration values from 3 to $100 \mathrm{~g}$. Acceleration of a shock pulse at which the operation of the pointing mechanism occurs is $91 \mathrm{~g}$ and $92 \mathrm{~g}$ for a serial relay for an experienced relay, and the power consumption of the relay is $0.107 \mathrm{~W}$ and $0.3 \mathrm{~W}$, respectively.

Thus, a significant reduction in power consumption has been achieved while maintaining the shock resistance of prototypes at the level of serial relays. 


\section{Findings}

1. Based on the analysis of the mechanisms of electromechanical devices for various purposes, we consider it expedient to classify in their classification by functional purpose a group of specific mechanisms with the common name "trigger devices of electromechanical devices", a distinctive feature of which is the mechanical characteristic due to the action of the return spring only.

2. Using the example of calculating the index relay, it is shown and experimentally confirmed that a change in the initial force of the mechanical characteristic and its rigidity leads to a decrease in power consumption while maintaining shock stability.

\section{References}

1. A.V. Gordon, A.G. Slivinskaja, DC electromagnets, 447 (1960)

2. GOST 19264-82 control electromagnets. General technical conditions.

3. M.A. Lyubchik, Optimal design of power electromagnetic mechanisms, 392 (1974)

4. HYUNDAI U-Series. Molded Case Circuit Breakers (ISCB) Technical Catalog 2014 [Electronic resource]. Access mode: http://hyundaielectric.com.ua/themes/hyundai/content/catalogs/pd f_pict/MCCB_U_rus.pdf.

5. A.D. Frolov, Theoretical bases of design and reliability of electronic equipment, 488 (1970) 